\title{
Modello processuale accusatorio e sovraccarico del sistema giudiziario
}

\section{"Accusatorial" model of procedure and overload of the judicial system}

\section{Modelo processual acusatório e sobrecarga do sistema judicial}

\section{Giulio Illuminati ${ }^{1}$}

LUISS “Guido Carli” Roma / Italia giulio.illuminati@unibo.it

https://orcid.org/0000-0002-6067-2859

\begin{abstract}
AвSTRACt: Lo scritto analizza i diversi modelli processuali penali, dedicando particolare attenzione alla crisi del sistema giudiziario italiano.

Parole-Chiave: sistema accusatorio; sistema inquisitorio; sistema misto; processo adversary; processo penale italiano.
\end{abstract}

ABSTRACT: The essay analyzes the different models of criminal procedure, devoting particular attention to the crisis of the Italian judicial system.

KeYwords: accusatorial system; inquisitorial system; mixed system; adversary proceeding; Italian criminal process.

Resumo: Este artigo analisa os diferentes modelos de processo penal, dedicando especial atenção à crise do sistema judicial italiano.

Palavras-chave: sistema acusatório; sistema inquisitório; sistema misto; processo adversarial; processo penal italiano.

SommaRIO: 1. II sistema accusatorio come tipo ideale. - 2. II valore storico della definizione - 3. Il sistema accusatorio dal punto di vista dogmatico. - 4. Il cosiddetto sistema misto. - 5. Caratteri comuni del diritto processuale penale moderno. - 6. II processo accusatorio in Italia. -

1 Già ordinario nell'Alma Mater Studiorum - Università di Bologna / Italia. Professore di Diritto processuale penale - LUISS “Guido Carli” Roma / Italia. 
7. Processo accusatorio e processo adversary. - 8. L'eccessiva durata del processo penale italiano: crisi del modello accusatorio. - 9. Alternative al processo penale per un recupero dell'efficienza del sistema. - 10. Conclusione.

1. Il sistema accusatorio come tipo ideale. - Anche se è largamente utilizzato per identificare un determinato modello processuale, il concetto di sistema accusatorio - come quello, ad esso contrapposto, di sistema inquisitorio - viene spesso definito in maniera molto diversa dagli studiosi. La distinzione finisce perciò col soffrire di una certa perdita di senso, tanto che talvolta viene considerata di puro interesse storico. Ciò dipende dal fatto che si tratta di concetti astratti, che rimandano alla elaborazione teorica di due sistemi ipotetici, ricavati, mediante generalizzazione, a partire da alcuni caratteri tipici degli ordinamenti processuali reali. Non è dunque in questione l'analisi e l'inquadramento sistematico di un fenomeno normativo, da studiare secondo i consueti strumenti dell'ermeneutica giuridica, quanto piuttosto la definizione di una scala di valori ideologicamente orientata. Infatti i lineamenti del sistema accusatorio si identificano solo per contrapposizione a quelli del sistema inquisitorio, e viceversa: l'uno e l'altro, dunque, rappresentano tipi ideali, collocati agli estremi di una linea continua all'interno della quale possono combinarsi secondo modalità differenti, in relazione a numerose variabili. In concreto, dunque, esistono sistemi processuali solo tendenzialmente definibili come accusatori o inquisitori, nessuno di essi essendo perfettamente corrispondente al tipo, nella forma cosiddetta "pura".

Dal punto di vista empirico, dunque, l'appartenenza di un determinato sistema processuale all'una o all'altra categoria è determinata da un esame comparativo, il cui risultato dipende da quegli aspetti specifici di volta in volta considerati essenziali all'integrazione del modello. Di solito viene aprioristicamente individuata una serie di principi generali che rappresentano la sintesi del sistema di riferimento, dai quali si deducono le conseguenze necessarie sul piano normativo. Tale ricostruzione tuttavia si basa su proposizioni valutative, dalle quali è delineato un "dover essere" del processo secondo orientamenti che sono determinati dal punto di vista soggettivo adottato. 
Pur con le cautele imposte dalla relatività della distinzione, è tuttavia necessario sottolineare che a ciascuno dei due modelli corrisponde dal punto di vista tecnico una diversa struttura, alla quale occorre fare riferimento se si vuole che abbia senso una classificazione che può ancora essere considerata utile come criterio di giudizio. A tale scopo tuttavia è necessario stabilire preliminarmente in che modo vanno isolati i caratteri che consentano di riconoscere un sistema come accusatorio o inquisitorio: quei caratteri, cioè, in assenza dei quali si dovrà escludere che un certo processo possa essere qualificato come appartenente al tipo.

2. Il valore storico della definizione. - La distinzione tra sistema accusatorio e sistema inquisitorio ha contemporaneamente un significato storico e un significato teorico-dogmatico, che non si implicano necessariamente a vicenda ${ }^{2}$. In altre parole, può accadere che alcuni tratti distintivi riscontrabili nei sistemi processuali classificati come accusatori o inquisitori, che si sono effettivamente succeduti nel tempo, non siano da considerare essenziali all'integrazione del modello teorico; oppure che non siano attualmente più significativi, perché ormai patrimonio comune degli ordinamenti moderni.

L’approccio storico, comunque, torna utile perché consente non solo di identificare le ascendenze dell'alternativa considerata, ma anche di comprendere appieno il significato dei parametri di riferimento della contrapposizione. La ricostruzione dogmatica, invece, anche se necessariamente convenzionale e quindi, come si è detto, sempre in certa misura opinabile, richiede la creazione di una struttura concettuale tecnicamente corretta, completa e logicamente coerente, rispetto alla quale l'accento viene posto principalmente sulle modalità di accertamento della responsabilità penale, e in particolare sui criteri di ammissione e acquisizione della prova.

Effettivamente si assiste, nel corso dei secoli, ad un avvicendamento dei due diversi sistemi. E' diventato quasi un luogo comune il riferimento al processo penale romano del periodo repubblicano come paradigma del sistema accusatorio e a quello imperiale classico per l'inquisitorio. La 2 L. Ferrajoli, Diritto e ragione. Teoria del garantismo penale, $8^{\text {a }}$ ed., Bari, 2004,
p. 574 s. 
contrapposizione, forse eccessivamente schematica, ha dovuto in parte la sua fortuna alle ideologie politiche volte alla promozione dei diritti individuali (che sono alla base delle moderne democrazie liberali), tendenti a mitizzare l'antica repubblica romana intesa come palladio delle libertà, contro la teoria dello stato assoluto di origine divina, simbolicamente rappresentato dal sopravvenire del principato. Tuttavia le informazioni pratiche che si possono ricavare da questa ricostruzione sono abbastanza modeste, soprattutto perché ogni paragone con il processo penale dell'età moderna resta in buona misura artificioso, mentre la ricerca di simmetrie appare alquanto forzata, essendo spesso dipesa, in passato, dalle strumentalizzazioni polemiche dei giuristi riformatori.

A ben considerare, la caratteristica saliente del diritto romano arcaico era l'incerta distinzione tra interesse privato e interesse pubblico alla giustizia penale, confusione ben rappresentata dalla originaria legittimazione della vendetta personale ${ }^{3}$. Ma anche con l'affermarsi della giurisdizione come funzione esclusiva dello Stato ${ }^{4}$ 'accusa rimaneva comunque riservata all'iniziativa privata: della parte offesa, ovvero di qualunque cittadino, in rappresentanza della società, quando fossero coinvolti direttamente interessi pubblici. Si comprende così come carattere tipico del processo accusatorio, sotto questo profilo, fosse tradizionalmente considerata la presenza, se non il monopolio, dell'accusa privata. Per contrasto, il processo inquisitorio si caratterizzerebbe come fondato sull'iniziativa dei funzionari pubblici: secondo il sistema - prevalente al tempo dell'impero - dell'azione penale esercitata anche d'ufficio da un magistrato delegato del principe, incaricato di scoprire i delitti e di trovarne le proves.

Un simile criterio di discriminazione fu effettivamente valido lungo tutto il medio evo, quando le due forme convivevano, fino al definitivo affermarsi della procedura inquisitoria ${ }^{6}$. Attualmente, però,

3 Per gli approfondimenti v. G. Pugliese, Processo privato e processo pubblico, in Riv. dir. proc., 1948, p. 72 s.; P. Fiorelli, Accusa e sistema accusatorio (diritto romano e intermedio), in Enc. dir., I, Milano, 1958, p. 330-331.

4 L. Ferrajoli, op. cit., p. 576.

5 P. Fiorelli, op. cit, p. 332.

$6 \quad$ P. Fiorelli, op. cit, p. 333. 
non sembra più significativo. La pubblicità dell'accusa è ormai un tratto ineliminabile di ogni ordinamento evoluto: anche là dove esiste la possibilità - prevalentemente residuale - di un'azione penale privata, il suo ruolo rimane in pratica del tutto marginale.

Neppure sembra risolutiva la versione aggiornata dello stesso criterio, con la quale si dà rilievo alla presenza di un apposito organo dell'accusa separato dal giudice. Se il cumulo delle funzioni denota inequivocabilmente un processo di tipo inquisitorio, poiché l'azione penale è esercitata dallo stesso soggetto destinato a valutare le prove e a decidere, è però vero che le due figure oggi risultano quasi ovunque formalmente distinte, salvo sporadiche eccezioni.

Ciò che veramente si può far risalire all'impero romano, per il tramite del diritto canonico medievale, è la tradizione burocratica, che ha contribuito alla progressiva burocratizzazione anche della funzione giurisdizionale. Si tratta di un'eredità culturale, fatta propria dagli Stati assoluti e perciò predominante nell'Europa continentale, che ancora produce i suoi effetti. Nemmeno la rivoluzione francese riuscì a sovvertirla in modo durevole: questa è probabilmente una delle ragioni che possono spiegare il sostanziale insuccesso, all'epoca, delle riforme processuali in senso accusatorio, imperniate viceversa su una larga partecipazione dei cittadini agli organi giudiziari e quindi sul cosiddetto giudizio dei pari.

3. Il sistema accusatorio dal punto di vista dogmatico. - Nell'accezione moderna, più significativa dal punto di vista dogmatico, la dicotomia si riferisce essenzialmente al metodo dell'accertamento giudiziale. La sua origine si può far risalire alle teorizzazioni dei philosophes illuministi, con il ripudio, in seguito alla rivoluzione francese, del processo inquisitorio dell'ancien régime. Il processo inquisitorio continentale, con le sue le tecniche scientificamente elaborate e il complesso armamentario delle prove legali, aveva infatti trovato la più importante sistemazione normativa nell'Ordonnance francese del $1670^{7}$. Si deve notare che viceversa nel common law inglese l'inquisizione - sebbene introdotta nel XV secolo dalla monarchia assoluta - era rimasta marginale e non era mai riuscita ad attecchire stabilmente, a vantaggio del processo davanti alla giuria

7 Ordonnance criminelle du mois d'août 1670 (26 agosto 1670). 
popolare: e proprio al sistema processuale inglese, anche se culturalmente meno evoluto (e certo all'epoca non assistito dalle garanzie che oggi conosciamo $)^{8}$, facevano riferimento i riformatori, anche sopravvalutandolo rispetto alla sua reale natura.

L'inquisizione consisteva in una partita a due tra l'accusato e il giudice-accusatore, senza una vera dialettica e perciò fortemente squilibrata a sfavore dell'imputato, che ne finiva inevitabilmente schiacciato'. L'inquisitore iniziava l'indagine segretamente e d'ufficio (o su denunzia anche anonima); i testimoni venivano sentiti in segreto senza alcuna partecipazione della difesa; l'imputato, senza poter conoscere con precisione l'addebito, né le prove a carico, veniva interrogato sotto giuramento ed eventualmente sottoposto a tortura per ottenerne la confessione, prova legale per eccellenza; testimonianze e interrogatorio erano documentati minuziosamente in verbali, che alla chiusura dell'inchiesta venivano trasmessi con tutti gli atti del processo al tribunale perché in base ad essi pronunciasse la sentenza.

Viceversa, il processo accusatorio anglosassone si svolgeva in un dibattimento pubblico, senza interrogatorio dell'imputato, con l'assunzione orale delle testimonianze, sottoposte alla valutazione immediata dalla giuria per l'emissione del verdetto ${ }^{10}$. Emergono così, per contrapposizione, i caratteri essenziali del sistema, ancora d'attualità nonostante i termini di riferimento siano oggi cambiati: pubblicità, oralità-immediatezza (che implica la percezione diretta della prova), contraddittorio: nonché, come logico corollario, libero convincimento del giudice.

Secondo l'opinione prevalente ${ }^{11}$, dunque, inquisitorio si può definire il metodo che esclude la dialettica tra accusa e difesa, per cui l'indagine, svolta unilateralmente dal magistrato, procede in modo analitico, potenzialmente senza limiti, e l'acquisizione delle prove è scritta e

8 L. Radzinovicz, A History of English Criminal Law and its Administration from 1750, I, London, 1948, p. 3 s.

9 Per una efficace sintesi v. F. Cordero, Procedura penale, $9^{\mathrm{a}}$ ed., Milano 2012, p. 21-38.

10 V. sul punto M. Ploscowe, The development of present-day criminal procedures in Europe and America, in 18 Harv. L. Rev., 1935, p. 453 s., 459-60.

11 Si veda, per tutti, la classificazione di G. Conso, Accusa e sistema accusatorio (diritto processuale penale), in Enc. dir., I, Milano 1958, p. 336. 
segreta; accusatorio, quello basato sul contraddittorio delle parti, sul tema proposto dall'accusa, davanti ad un giudice non destinato alla ricerca delle prove, nella tendenziale unità di tempo rappresentata dal dibattimento orale e pubblico.

Come s'è accennato, sono individuabili ulteriori connotazioni, ma solo come aspetti eventuali che costituiscono un'eredità storica, ovvero come conseguenze favorite naturalmente dalla scelta metodologica: così, ad esempio, la presenza della giuria, ovvero l'esame incrociato dei testimoni gestito direttamente dalle parti. Si annovera di solito tra i caratteri distintivi anche il regime della libertà personale dell'imputato, poiché viene considerata tipica del sistema inquisitorio la carcerazione preventiva: l'assunto è sostenibile solo in quanto la misura sia considerata strumentale all'indagine segreta e all'interrogatorio come fonte di prova: sotto altri profili, invece, la coercizione personale non sembra sostanzialmente condizionata - a parte i presupposti specifici e le modalità, che possono variare - dalla natura accusatoria o inquisitoria del processo.

Dalle grandi linee escono con sufficiente determinatezza due modelli culturali. Al fondo si può tuttora leggere un diverso modo di intendere i rapporti tra il cittadino e l'autorità dello Stato: tanto che non è sbagliata, per quanto schematica, la ricorrente affermazione secondo cui il processo accusatorio sarebbe tendenzialmente espressione dei regimi democratici, quello inquisitorio dei regimi autoritari ${ }^{12}$.

4. Il cosiddetto sistema misto. - Sistema "misto" viene definito quello adottato dagli ordinamenti processuali dell'Europa continentale a partire dal code d'instruction criminelle napoleonico del 1808. Tuttavia, ove si prescinda dal valore convenzionale consacrato dall'uso, la formula appare priva di un significato autonomo, anzi, finisce con l'impedire un'esatta classificazione. Non è possibile infatti determinare i caratteri distintivi del processo misto come tale, senza rinviare alle peculiarità dei singoli casi. Se è vero, come si è osservato, che col nome di sistema accusatorio e di sistema inquisitorio si designano due tipi ideali contrapposti, qualunque scostamento dal tipo dà luogo ad un processo misto, indipendentemente dal modo in cui gli elementi dei due sistemi sono combinati.

12 Vedi ad esempio L. Lucchini, Elementi di procedura penale, Firenze, 1895, p. 19. 
Secondo l'opinione tramandata, il cosiddetto processo misto sarebbe un'opportuna mediazione tra l'accusatorio e l'inquisitorio, con lo scopo di contemperare le esigenze di difesa della società e la tutela dei diritti individuali, messe in pericolo, rispettivamente, dall'adesione all'uno o all'altro sistema. La struttura dialettica del primo viene perciò inserita su un'indagine compiuta con i metodi del secondo. Il risultato è un processo in due fasi: il giudizio, pubblico e orale, si svolge in contraddittorio sul tema prefissato dopo un'istruzione scritta e segreta, condotta dal magistrato, senza la partecipazione della difesa, con pieni poteri di accertamento in ogni direzione.

Lo sdoppiamento così realizzato, nel tentativo di conciliare modelli opposti, finisce col mancare di coerenza sistematica: ed in effetti questo tipo di critica venne subito formulata, insieme all'addebito di sommare i difetti, e non i vantaggi, dei due sistemi ${ }^{13}$. Tuttavia - anche senza accedere all'opinione di chi ritiene questo processo un tertium genus, né accusatorio, né inquisitorio (né "misto") ${ }^{14}$ - la soluzione non è priva di tratti originali, e sarebbe un errore negarle una precisa identità. Tutto dipende, comunque, dal modo in cui sono disciplinati i rapporti tra la fase dell'istruzione e quella del giudizio: la fisionomia del processo cambia completamente a seconda che le risultanze istruttorie abbiano valore determinante, ovvero le prove valutabili ai fini della decisione possano essere acquisite solo nel contraddittorio dibattimentale.

Da questo punto di vista, il sistema francese e i suoi derivati (come l'italiano prima della riforma) sono fondamentalmente da considerare un'evoluzione, con i dovuti aggiornamenti, del vecchio processo inquisitorio. Infatti, dopo le prime esperienze della legislazione rivoluzionaria, intesa a trasformare in modo radicale il precedente ordinamento con l'adozione di schemi accusatori, la fase del dibattimento andò progressivamente perdendo peso a vantaggio dell'istruzione - più consona alle esigenze del mutato clima politico - fino a ridursi a mero controllo estrinseco delle conclusioni già raggiunte dal giudice istruttore, se non addirittura ad un'inutile messa in scena.

13 G. Carmignani, Teoria delle leggi della sicurezza sociale, Pisa, 1832, IV, $46 \mathrm{~s}$.

14 G. Foschini, Sistema del diritto processuale penale, I, Milano, 1965, $226 \mathrm{~s}$. 
Il progressivo potenziamento, in epoca più recente, dei diritti della difesa è valso ad attenuare gli aspetti più marcatamente autoritari di un simile assetto processuale; ma la struttura tendenzialmente inquisitoria è destinata a sopravvivere immutata finché il contraddittorio continua ad essere escluso dai principali atti di acquisizione probatoria compiuti dal magistrato nell'istruzione e i verbali che li documentano sono utilizzabili nel giudizio, risultando il più delle volte decisivi per il suo esito.

\section{Caratteri comuni del diritto processuale penale moderno - Negli} ultimi decenni, tuttavia, la distanza tra i due modelli contrapposti si è in parte ridotta, almeno per quanto concerne gli aspetti più evidenti. Ciò è dovuto soprattutto alla necessità di dare attuazione alle garanzie del giusto processo prescritte dalle carte costituzionali o dalle convenzioni internazionali sui diritti dell'uomo. Si può osservare come nelle fonti di questo livello ad essere affermati siano i tradizionali diritti della persona di origine illuministica: la coesistenza di tali direttive con un sistema inquisitorio nel senso tradizionale del termine sarebbe inconcepibile, mentre al contrario risultano valorizzati alcuni degli aspetti caratteristici del sistema accusatorio. Resta però il fatto che non è riconoscibile un'opzione precisa a favore dell'uno o dell'altro sistema, dato che le norme in questione appaiono il più delle volte formulate in maniera volutamente generica, aperta ad un'interpretazione adeguatrice, proprio per consentire la loro applicazione a prescindere dal contesto ordinamentale nel quale sono destinate ad operare.

Si può dunque affermare che taluni principi fondamentali, come la pubblicità delle udienze, l'esposizione delle ragioni in contraddittorio, l'inviolabilità della difesa, l'imparzialità del giudice, la presunzione d'innocenza dell'accusato, debbano darsi per acquisiti, se non altro come criteri direttivi tendenziali, nelle società democratiche, in ogni tipo di processo, anche se ciò non implica necessariamente l'adesione ad un modello ideale accusatorio. Interessante, sotto questo profilo, l'esperienza della Corte penale internazionale ${ }^{15}$ - che vuole fungere da esempio universale di giustizia amministrata nel pieno rispetto dei diritti della persona - nel

15 K. Ambos, International criminal procedure: "adversarial", "inquisitorial" or mixed?, in Int'l. Crim. L. Rev., 2003, p. 1 s. 
cui ordinamento si tende a conciliare l'oralità e il contraddittorio dibattimentale fra le parti con i poteri discrezionali del giudice nella provvista del materiale probatorio da utilizzare ${ }^{16}$.

Come criterio globale di comprensione della realtà, dunque, l'alternativa di cui ci occupiamo non sembra più di per sé sola sufficiente. Altre chiavi di lettura possono essere impiegate quando l'analisi del processo riguarda particolarmente il grado di attuazione delle garanzie fondamentali. Ciò nondimeno, non si può affermare che il modello processuale prescelto sia indifferente rispetto all'obiettivo di tutelare i diritti dell'accusato. Di fatto esso determina gli strumenti utilizzabili, e perciò il livello di effettività della tutela. Così, per fare qualche esempio, appartiene alla logica inquisitoria riconoscere il diritto di difesa come controllo a posteriori, mediante il regime delle nullità e il favore per le impugnazioni, a quella accusatoria permettere al difensore il massimo di partecipazione agli atti del processo, con la possibilità di controllarne immediatamente la correttezza; alla prima, perseguire una decisione imparziale assegnando anche alla pubblica accusa una funzione quasi-giudiziale, alla seconda, assicurare l'imparzialità accentuando la separazione di ruoli tra il giudice e le parti.

6. Il processo accusatorio in Italia. - La delega al governo per la riforma del codice di procedura penale italiano (l. 16 febbraio 1987, n. 81 ), risultato di una più che ventennale elaborazione, segna il passaggio radicale dal sistema misto di stampo inquisitorio al sistema accusatorio: tale scelta è esplicitamente enunciata nella premessa all'art. 2, il quale è dedicato ai principi e criteri direttivi. Da questi si desume che carattere essenziale del sistema è considerato il principio secondo cui la prova va formata davanti al giudice del giudizio nel contraddittorio delle parti. $\mathrm{Ne}$ segue la distinzione tra la fase delle indagini e quella del dibattimento, poiché le informazioni raccolte nelle indagini preliminari non sono idonee ad essere utilizzate per la decisione finale: essa può legittimamente fondarsi soltanto sulle prove formate nella pubblica udienza rispettando il principio di oralità-immediatezza.

16 Per ulteriori approfondimenti v. M. Caianiello, Disclosure before the ICC. The emergence of a new form of policies implementation system in international criminal justice?, in Int'l Crim. L. Rev., 2010, p. 23 s. 
Secondo questa prospettiva, le indagini preliminari sono attribuite al pubblico ministero, con l'ausilio della polizia giudiziaria, ma solo per decidere sull'esercizio dell'azione penale e la preparazione dell'accusa per il giudizio: i verbali delle dichiarazioni acquisite a questi fini non sono di regola leggibili nel dibattimento, se non per contestare la credibilità del dichiarante nel corso dell'esame. Una delle innovazioni più significative è il cosiddetto "doppio fascicolo", mirante ad evitare che le risultanze documentate delle indagini possano condizionare il convincimento del giudice. Al giudice vengono trasmessi, con il "fascicolo per il dibattimento", soltanto quei pochi atti che gli è consentito conoscere, vale a dire principalmente gli atti irripetibili, le prove non rinviabili acquisite anticipatamente in contraddittorio, i documenti utili per il giudizio sulla personalità dell'imputato, oltre agli atti riguardanti la corretta insaturazione del giudizio. Gli altri atti di indagine restano nel "fascicolo del pubblico ministero", che è conosciuto solo dalle parti, e possono essere trasferiti nel fascicolo per il dibattimento e utilizzati per la decisione solo in casi eccezionali, ad esempio se il testimone è stato minacciato o subornato, o se l'esame sia divenuto impossibile, o se c'è l'accordo delle parti ${ }^{17}$.

In sintesi, si può affermare che il processo accusatorio elaborato dal codice di procedura penale si basa sulla separazione delle funzioni e sulla separazione delle fasi. La funzione del giudice che deve decidere il merito deve essere separata da quella degli organi di investigazione, dal momento che il compito di ricercare e introdurre le prove non può essere attribuito allo stesso soggetto destinato a valutarle, che rischia altrimenti di perdere la necessaria equidistanza dalle parti. La separazione della fase delle indagini da quella del giudizio serve ad assicurare l'effettiva attuazione del contraddittorio, privilegiando inoltre il contatto diretto del giudice con la prova rispetto all'utilizzazione dei verbali di atti compiuti fuori dal dibattimento. Lo strumento prescelto per la formazione della prova in ottemperanza ai suddetti criteri è l'esame diretto ed incrociato dei testimoni che sono presentati dalle parti, con attribuzione al giudice di poteri solo residuali di interloquire nell'esame, ovvero, in caso di assoluta necessità, di supplire all'inerzia delle parti ammettendo prove d'ufficio.

17 Per un'esposizione più dettagliata degli aspetti specifici e delle vicende successive della riforma del processo penale si rinvia a G. Illuminati, op. cit., p. 148 s. 
7. Processo accusatorio e processo adversary. - Il processo penale adversary di stampo anglo-americano è stato da sempre considerato il paradigma tipico del sistema accusatorio, ed effettivamente ha rappresentato il modello al quale sotto molti profili anche il legislatore italiano si è ispirato. Esso presenta tuttavia numerose peculiarità, che spesso non sono suscettibili di essere trapiantate in un ordinamento diverso, o che non risulterebbero compatibili con i suoi principi generali informatori o con le norme costituzionali.

L'aspetto più caratteristico è il potere delle parti di disporre non solo delle prove da presentare in giudizio, ma anche dell'oggetto stesso del procedimento.

La passività del giudice, come corrispettivo dell'iniziativa riservata alle parti, è considerata essenziale (anche se in teoria alcuni poteri d'ufficio gli sarebbero riconosciuti). Il giudice ha solo il ruolo di garantire il rispetto delle regole ed è perciò considerato un semplice arbitro della contesa: anche perché non è gravato della funzione di accertare i fatti, spettante esclusivamente alla giuria. Il processo penale è infatti concepito come strumento di composizione dei conflitti, concezione estranea agli ordinamenti dell'Europa continentale, che attribuiscono alla giustizia penale la funzione di realizzare le scelte di politica criminale dello Stato ${ }^{18}$.

Stando alla definizione che ne abbiamo dato in precedenza, comunque, questi aspetti specifici non sembrano essenziali per l'integrazione del modello accusatorio. Non si può cioè affermare una coincidenza fra sistema accusatorio e adversary system.

Il processo accusatorio italiano, in particolare, si discosta sensibilmente da quello tipico dei paesi di lingua inglese.

Una differenza significativa è rappresentata dall'assenza della giuria popolare. Il giudizio si svolge per lo più davanti a giudici professionali: e anche quando - per i reati più gravi - è prevista la partecipazione di giudici popolari, è istituito un collegio misto, la corte d'assise, composto da due giudici togati e sei laici, che decidono insieme, a maggioranza, tutte le questioni di fatto e di diritto. Anche le regole di ammissione e di esclusione della prova cambiano di conseguenza: non

18 In argomento cfr. M.R. Damaška, Evidence Law Adrift, New Haven-London, 1997, trad. it., Il diritto delle prove alla deriva, Bologna, 2003, p. 113 s. 
esiste la prevalenza assoluta della prova orale su quella documentale, e non occorre tutelare preventivamente una giuria inesperta di diritto dalla suggestione delle prove invalide o di quelle la cui inaffidabilità può superarne il valore probatorio. Inoltre la motivazione, doverosa per la sentenza del tribunale o della corte d'assise e assente nel verdetto della giuria, consente di verificare l'osservanza da parte del giudice dei divieti di utilizzazione e dunque non impone di escludere materialmente la prova inutilizzabile.

A livello costituzionale, poi, assume un rilievo determinante l'obbligatorietà dell'azione penale (art. 112 Cost.), che richiede un costante controllo del giudice sull'iniziativa del pubblico ministero. La responsabilità dell'attuazione del principio di legalità, con l'applicazione della sanzione penale, risulta pertanto condivisa tra il pubblico ministero e il giudice, che non è vincolato dalle richieste dell'accusa: il giudice può condannare anche se il pubblico ministero chiede l'assoluzione, può applicare una pena superiore a quella richiesta, può modificare il titolo del reato indicato nell'imputazione. Viceversa, nel processo adversary, il prosecutor ha il controllo esclusivo sull'azione penale e può astenersi dall'esercitarla, esercitarla per un reato minore o diverso da quello risultante dalle indagini, e anche lasciarla cadere nel corso del processo.

Questa dinamica si riflette anche sui procedimenti speciali basati sull'accordo delle parti, destinati ad evitare il dibattimento. Nell'ordinamento italiano, che pure ha introdotto l'applicazione della pena su richiesta delle parti (insieme ad altre forme consensuali di semplificazione come il giudizio abbreviato), l'obbligatorietà dell'azione penale impedisce una vera e propria transazione sull'accusa: ciò comporta in ogni caso una valutazione di merito da parte del giudice ed un controllo sui contenuti dell'accordo tra le parti, in relazione al principio di legalità. Occorre sottolineare che nemmeno questo modo alternativo di procedere, con rinuncia al giudizio in contraddittorio, va considerato un aspetto essenziale per caratterizzare il sistema in senso accusatorio, sebbene sia impiegato con la massima larghezza negli ordinamenti inglese e americano. Si può anzi affermare che si tratta di una soluzione di tipo inquisitorio ${ }^{19}$, anche se legittimata dal consenso dell'imputato.

19 A. Nappi, Guida al codice di procedura penale, 10 ed., Milano, 2007, p. 13 s. 


\section{L'eccessiva durata del processo penale italiano: crisi del modello}

accusatorio. - Com'è noto, il processo accusatorio è molto dispendioso in termini di tempo e risorse necessarie per il suo corretto svolgimento. Se tutte le prove vanno acquisite oralmente davanti al giudice con la tecnica dell'esame diretto e incrociato ad opera delle parti, e - corrispondentemente - le dichiarazioni raccolte dal pubblico ministero e dalla polizia nel corso delle indagini (non importa quanto lunghe e complesse) debbono essere tutte ripetute in dibattimento, perché di regola non se ne può tener conto, è evidente che risulta possibile celebrare e concludere definitivamente soltanto un numero limitato di processi, con la conseguenza che il carico degli uffici giudiziari inevitabilmente tende ad aumentare, restando in buona parte impossibile da smaltire in tempi brevi. Ciò comporta, in Italia, un'eccessiva durata del giudizio dibattimentale, che deve perciò necessariamente svolgersi nell'arco di numerose udienze, il più delle volte molto distanziate fra loro.

La sproporzione tra il numero di processi in attesa di essere celebrati e le capacità che il sistema ha di gestirli impone ritardi non solo nell'effettiva durata dei dibattimenti, ma anche nel tempo necessario per la loro instaurazione, ad indagini ormai concluse, quando è il momento di iscriverli nel ruolo di udienza del tribunale. Se a questo si aggiungono i tempi dei due gradi di impugnazione, l'accesso ai quali è consentito praticamente senza limiti, e che soffrono dei medesimi problemi di sovraccarico, non c'è da stupirsi se per arrivare ad una sentenza definitiva bisogna attendere un termine tutt'altro che ragionevole, a dispetto del principio solennemente enunciato dall'art 111 della Costituzione italiana, oltre che dalle Carte internazionali dei diritti umani.

Al riguardo, non si può fare a meno di considerare che nel sistema inglese e in quello degli Stati Uniti il numero di casi che vengono effettivamente trattati davanti alla giuria, seguendo le regole ordinarie del processo adversary, non supera il dieci per cento del totale, dato che tutti gli altri si concludono per lo più con un'ammissione di colpevolezza dell'imputato, spesso negoziata con l'accusa. Solo così è possibile assicurare l'efficienza complessiva della giustizia penale.

Nelle giurisdizioni degli Stati Uniti la celerità del processo risulta formalmente garantita - in caso di violazione del diritto costituzionale allo speedy trial (VI emendamento) - dal drastico rimedio del dismissal, 
equivalente all'archiviazione dell'accusa ma preclusivo della possibilità di una successiva riapertura del procedimento. Senza entrare nei dettagli, si può ricordare che nella giurisdizione federale e in quelle statali la durata media di un processo, fino alla sentenza di primo grado, si aggira sui sei-sette mesi $^{20}$, e non è considerata ragionevole, salvo motivi particolari, una durata superiore ad un anno. Occorre aggiungere che la sentenza non è appellabile nel merito, essendo proponibili solo questioni di diritto, e che l'accesso ad un ulteriore grado di impugnazione non è garantito in ogni caso, perché il ricorso è soggetto ad una valutazione preliminare della corte adita, che può decidere di non ammetterlo se non ritiene opportuno occuparsene. Ciò riduce sensibilmente i tempi complessivi e disincentiva impugnazioni meramente dilatorie.

Si deve tenere presente che è la struttura stessa del processo accusatorio, in quanto basato sul principio di oralità e immediatezza nell'assunzione delle prove, a suggerire la celebrazione del processo in tempi stretti, così da garantire la reperibilità dei testimoni e soprattutto l'affidabilità del loro ricordo, dando al tempo stesso alle parti la possibilità di verificare tramite l'esame incrociato la correttezza della percezione dei fatti e della loro rappresentazione. Una testimonianza resa a molta distanza dall'evento difficilmente potrà descriverne gli elementi rilevanti in maniera puntuale e oggettiva, e si limiterà a rievocare la ricostruzione mentale che si è venuta a fissare nella memoria del testimone col passare del tempo.

Il processo con giuria, poi, impone in maniera ancor più evidente la massima concentrazione del giudizio, mal conciliandosi con lunghi rinvii delle udienze, posto che la decisione si basa su quanto visto e udito direttamente dai giurati e dunque su quello che ricordano dell'intero dibattimento. La concentrazione è perciò anche interesse delle parti, che devono riuscire a sintetizzare al massimo la presentazione delle prove, in modo da colpire efficacemente l'attenzione della giuria.

Va però anche considerato che il modello adversary si basa essenzialmente sul confronto fra le parti contrapposte, inteso come metodo più

20 V. Fanchiotti, The right to a speedy trial nell'esperienza nordamericana, in AA. VV., La ragionevole durata del processo. Garanzie ed efficienza della giustizia penale, a cura di R.E. Kostoris, Torino, 2005, p. 13 s. 
efficace per accertare i fatti e non lasciare aspetti inesplorati. Ne deriva un accentuato livello di conflittualità tra accusa e difesa, che inevitabilmente favorisce gli imputati più forti e meglio assistiti. La discriminazione tra abbienti e non abbienti si può riscontrare in tutti gli ordinamenti processuali ed è in buona misura ineliminabile: nei sistemi di cui stiamo parlando, tuttavia, diventa un ulteriore potente strumento di semplificazione e di accelerazione. Dal momento che solo pochi imputati possono permettersi di sostenere gli elevatissimi costi di un processo con giuria, assumendosi inoltre il rischio di una condanna molto più pesante, il ricorso al plea bargaining, con la conseguente ammissione di colpevolezza che esclude il giudizio, è frequentissimo, e ciò anche a prescindere dalla effettiva fondatezza dell'accusa.

Anche il sistema di assistenza giudiziaria ai non abbienti porta per forza di cose a privilegiare la celerità, poiché le risorse sono naturalmente limitate e i difensori hanno interesse ad una semplice e rapida conclusione dei processi. Si tratta infatti di pubblici funzionari che devono massimizzare le risorse disponibili, come negli Stati Uniti dove esiste la figura del public defender, ovvero di avvocati privati pagati dallo Stato, come in Inghilterra, che hanno la necessità di portare avanti molte difese in breve tempo per guadagnare a sufficienza ${ }^{21}$. In ogni caso, poi, rimane fuori dall'assistenza un'ampia fascia della popolazione, titolare di un reddito che non consente l'accesso alla difesa fornita dallo Stato, ma che tuttavia non ha i mezzi per pagare le spese e gli onorari di un avvocato per il giudizio.

Sotto il profilo dell'equità, la pratica del plea bargaining è da tempo, per evidenti motivi, criticata dagli studiosi ${ }^{22}$. Occorre domandarsi se si tratti di una contropartita adeguata rispetto alla necessità di assicurare l'efficienza complessiva del sistema giudiziario, e se sia socialmente accettabile una così marcata discriminazione.

21 D. Nelken, La durata del processo penale in prospettiva empirica: riforme giuridiche e contesti sociali, in AA.VV., La ragionevole durata, cit., p. 28.

22 V. ad esempio J. H. Langbein, Torture and plea bargaining, in 46 Univ. Chicago L. Rev., 1978-79, p. 3 s. Per un'analisi critica in chiave comparatistica, v. R. Van Cleave, An offer you can't refuse? Punishment without trial in Italy and the United States: the search for truth and an efficient criminal justice system, in 11 Emory Int'l L. Rev., 1997, p. 419 s. 
Non si può nascondere, comunque, che le disuguaglianze determinate dalle condizioni economiche hanno conseguenze altrettanto pesanti nei sistemi in cui la giustizia è lenta e inefficiente e il numero dei processi da celebrare è eccessivamente alto. L'imputato ricco o potente, tecnicamente ben attrezzato, ha la possibilità di sfruttare numerose opportunità per ostacolare lo svolgimento del processo e rallentarlo, traendo il massimo vantaggio dal malfunzionamento delle strutture e dai ritardi fisiologici o patologici, fino ad arrivare non di rado ad assicurarsi una sostanziale impunità a causa dell'estinzione del reato.

\section{Alternative al processo penale per un recupero dell'efficienza}

del sistema. - Come si è già detto, il principio di legalità, espresso nella Costituzione italiana come obbligo per il pubblico ministero di esercitare l'azione penale, differenzia in maniera netta il nostro processo accusatorio da quello adversary dei paesi di common law, che si caratterizza per la discrezionalità del prosecutor, il quale può non formulare l'accusa, ritrattarla e anche deciderne autonomamente la qualificazione giuridica. Ciò naturalmente comporta la possibilità di selezionare i casi da sottoporre a processo e di commisurare il carico di lavoro alle effettive possibilità dell'ufficio, anche in ragione delle scelte di politica criminale.

Non si può nemmeno affermare, però, che l'azione penale discrezionale sia un carattere essenziale del modello accusatorio, anche se indubbiamente è in grado di assicurare una maggiore efficienza, considerati anche i già segnalati costi di questo tipo di processo in termini di tempo e di risorse necessarie. L'obbligatorietà dell'azione penale ha, almeno in linea di principio, la funzione di evitare disparità di trattamento fra i cittadini, e al tempo stesso tutela l'indipendenza del pubblico ministero da interferenze del potere esecutivo o di altri poteri. La sua centralità come strumento per l'attuazione della legalità nel campo penale è stata espressamente proclamata dalla Corte costituzionale italiana ${ }^{23}$. D'altra parte un organo dell'accusa al quale sia affidato il compito di decidere discrezionalmente quali reati perseguire e quali trascurare dovrebbe necessariamente risponderne politicamente: o direttamente ai cittadini quale ufficio elettivo, secondo il sistema statunitense, ovvero, come

23 Corte cost. 15 febbraio 1991, n. 88. 
avviene in molti altri paesi, al governo, a sua volta responsabile verso gli elettori. Verrebbe meno perciò l'indipendenza del pubblico ministero che caratterizza l'ordinamento italiano.

In un sistema ad azione penale obbligatoria risalta in maniera particolarmente evidente la sproporzione tra le risorse disponibili e quelle che sarebbero necessarie. Il problema non è peculiarmente italiano, ma in Italia è aggravato dalla qualità e dalla quantità molto bassa degli investimenti in materia di giustizia. Senza entrare nei dettagli, non sembra però verosimile prevedere un'inversione di tendenza, soprattutto in tempi di crisi economica: occorre pertanto verificare come sia possibile, agendo sul momento introduttivo del processo, razionalizzarne il funzionamento, in osservanza del principio della durata ragionevole.

Anche a prescindere dalle ragioni che suggeriscono di salvaguardare il principio di obbligatorietà, non è però detto che la discrezionalità dell'azione penale sia di per sé la soluzione. Il ricorso a criteri di opportunità non produce necessariamente un aumento di efficienza del sistema, perché in pratica, quali che siano le scelte operate, non si può pensare che ciò comporterebbe un'automatica riduzione della domanda di giustizia, destinata a restare in larga parte inevasa. Un sistema basato esclusivamente sulla produttività, in termini di rapporto costi-benefici - per quanto questo dato sia importante - conduce anche ad esiti consapevolmente iniqui.

Si deve allora cercare di riequilibrare la proporzione tra i casi in cui si rende necessario ricorrere al processo penale e la capacità di risposta del sistema. Soluzioni miracolose o di breve periodo non ne esistono: è la giustizia penale nel suo insieme che va ripensata.

Da tempo la dottrina più attenta evidenziava la necessità di procedere ad un'ampia depenalizzazione e all'aumento del numero dei reati perseguibili a querela di parte; nonché, contemporaneamente, alla diminuzione delle ipotesi di sanzione privativa della libertà personale, per la cui applicazione la tutela giurisdizionale è imprescindibile. L'osservazione può sembrare scontata, ma ci sono sempre stati ostacoli ideologici o pratici ad affrontare il problema in maniera radicale. E' noto a tutti che nel diritto italiano c'è un eccesso di penalizzazione, e che spesso la sanzione penale viene introdotta, a prescindere dalla sua concreta effettività, quando si vuole mandare un segnale all'opinione pubblica, 
anche per ragioni di propaganda politica. Esiste un numero smisurato di fattispecie incriminatrici che dovrebbero essere del tutto cancellate, o quanto meno affrontate con sanzioni non penali o con semplici obblighi di risarcimento o riparazione, evitando il ricorso ad una macchina così pesante e complessa - proprio perché destinata a garantire i diritti fondamentali - qual è il processo penale. Altrimenti si attiva una procedura ad alto tasso di garanzia anche per questioni di rilevanza minima, per le quali, il più delle volte, non si arriva nemmeno all'esecuzione di una condanna, col risultato che assai spesso il processo penale ha esclusivamente un'efficacia stigmatizzante.

In questa direzione ha di recente cominciato ad avviarsi il legislatore, con i decreti legislativi n. 7 e 8 del 15 gennaio $2016^{24}$, che hanno depenalizzato numerose fattispecie ormai obsolete o inutili e alcune categorie di illeciti minori. I reati depenalizzati prevedono ora solo sanzioni amministrative, oppure una riparazione pecuniaria in sede civile, che si aggiunge al risarcimento dei danni. Da ultimo, poi, con il d.lgs. 10 aprile 2018 , n. $36^{25}$, sono stati aumentati i casi di procedibilità a querela di parte, consentendo fra l'altro di applicare, quando la querela sia proposta, la nuova causa di estinzione del reato per condotte riparatorie, operante senza il consenso della persona offesa, di cui all'art. 162-ter c.p., inserito dalla stessa legge n. 103 del 2017.

Sarebbe inoltre indispensabile introdurre nell'esercizio dell'azione penale criteri di discrezionalità controllata, che come tali, secondo l'opinione più diffusa, non entrano necessariamente in conflitto col principio di obbligatorietà ${ }^{26}$, se ragionevoli e adeguatamente tipizzati. Non c'è dubbio che dietro l'obbligatorietà dell'azione penale si celi attualmente una discrezionalità di fatto, dato che gli uffici del pubblico ministero sono

24 In attuazione della delega contenuta nella 1. 28 aprile 2014, n. 67.

25 In attuazione della delega contenuta nella 1. 23 giugno 2017, n.103.

26 In questo senso v. già M. Chiavario, Ancora sull'azione penale obbligatoria: il principio e la realtà, in Id., L'azione penale tra diritto e politica, Padova, 1995, p. 131. Più di recente v., anche per ulteriori indicazioni, A. Ciavola, Il contributo della giustizia consensuale e riparativa all'efficienza dei modelli di giurisdizione, Torino, 2010, p. 95 s. La stessa Corte costituzionale, nella citata sentenza n. 88 del 1991, aveva escluso che l'obbligatorietà dell'azione penale significhi consequenzialità automatica tra notizia di reato e processo. 
comunque tenuti ad operare delle scelte di priorità nella trattazione delle notizie di reato, non essendo materialmente possibile gestirle tutte allo stesso modo. Quello che si dovrebbe pretendere è che queste scelte siano trasparenti e prevedibili.

Il modo migliore per assicurarlo sarebbe la predeterminazione legale dei presupposti in presenza dei quali, anche quando esistono elementi idonei a sostenere l'accusa, il pubblico ministero possa astenersi dall'agire, previa supervisione del giudice destinata a verificarne la corretta applicazione nel caso concreto ${ }^{27}$. Si dovrebbe cioè trovare un contemperamento fra opportunità e doverosità dell'azione: e la risposta penale può anche essere diversificata, senza violare le garanzie fondamentali, a seconda che si tratti di piccola o di grande criminalità ${ }^{28}$.

In una simile prospettiva, persegue l'obiettivo di alleggerire il carico giudiziario la nuova disciplina del proscioglimento per «particolare tenuità del fatto» ${ }^{29}$, anche se da taluno criticata in rapporto ai principi tradizionali del diritto penale sostanziale ${ }^{30}$. L'art. 131-bis c.p. prevede

27 D. Vicoli, Scelte del pubblico ministero nella trattazione delle notizie di reato e art. 112 Cost.: un tentativo di razionalizzazione, in Riv. it. dir. proc. pen., 2003, p. $251 \mathrm{~s}$.

28 A. Ciavola, op. cit., p. $101 \mathrm{~s}$.

29 Artt. 1-5 d.lgs. 16 marzo 2015, n. 28, approvato in attuazione della direttiva di legge delega contenuta nella già citata legge n. 67 del 2014. Il Governo era invitato $\mathrm{a}$ «escludere la punibilità di condotte sanzionate con la sola pena pecuniaria o con pene detentive non superiori nel massimo a cinque anni, quando risulti la particolare tenuità dell'offesa e la non abitualità del comportamento, senza pregiudizio per l'esercizio dell'azione civile per il risarcimento del danno e adeguando la relativa normativa processuale penale» (art. 1 comma 1 lett. $\mathrm{m}$ ).

Le premesse concettuali della novella risultano tuttavia largamente condivise. Il proscioglimento - che presuppone la sussistenza di un fatto tipico di reato, di modesta portata lesiva ma non totalmente inoffensivo (C.F. Grosso, La non punibilità per particolare tenuità del fatto, in Dir. pen. proc., 2015, p. 517) - risponde a due fondamentali esigenze di rango costituzionale: il principio di proporzionalità dell'intervento repressivo penale, che vieta, in ossequio al canone dell'ultima ratio, di sanzionare penalmente condotte che appaiano immeritevoli di pena nel caso specifico, ed anzi rende la declaratoria di tenuità del fatto una vera e propria «necessità di giustizia» in considerazione della «ormai acquisita consapevolezza dogmatica dei limiti della tipicità penale» (così F. Palazzo, Nel dedalo delle riforme prossime e venture, in Riv. it. dir. e proc. pen., 2014, p. 1706 s.); nonché l'esigenza di alleggerire il carico giudiziario e di restituire effettività al principio di obbligatorietà dell'azione penale (C.F. Grosso, op. loc. cit.). 
che se il reato è punito con pena detentiva non superiore nel massimo a cinque anni l'offesa può, a determinate condizioni, essere ritenuta non punibile. La non punibilità può essere dichiarata anche in sede di archiviazione della notizia di reato, su richiesta del pubblico ministero (art. 411 c.p.p.), o con una sentenza di proscioglimento predibattimentale (art. 469 comma 1-bis c.p.p.). In ogni caso i provvedimenti che dichiarano la non punibilità vengono iscritti nel casellario giudiziale e, se si tratta di sentenza pronunciata nel dibattimento, essa fa stato - quanto all'accertamento del fatto, della sua illiceità e della responsabilità dell'imputato - nel processo civile o amministrativo di danno promosso contro il prosciolto.

10. Conclusioni. - La scelta accusatoria del codice del 1988, da molti inizialmente osteggiata, è ormai da considerare irreversibile, anche se non ha portato ai risultati sperati quanto alla funzionalità del processo, non riuscendo ad assicurarne la ragionevole durata. Ciò si ripercuote, come appare evidente, sulla effettiva realizzazione del modello, che per attuare l'oralità-immediatezza esige la concentrazione del dibattimento. I lunghi rinvii delle udienze tradiscono lo spirito del sistema accusatorio, dal momento che il giudice finisce col dover valutare non tanto le prove percepite direttamente, quanto piuttosto il verbale dibattimentale che le documenta. Tuttavia un ritorno al vecchio sistema è impensabile, poiché non porterebbe alcun giovamento e rappresenterebbe solo un regresso in termini di garanzie.

Un altro dato ormai acquisito, e si può dire universalmente riconosciuto, è la necessità che siano contemplati percorsi differenziati, non solo all'interno del processo, mediante la deviazione sui riti alternativi, ma anche verso l'esterno, con una vera e propria "deprocessualizzazione" che consenta epiloghi di altro genere.

I procedimenti speciali come l'applicazione della pena su richiesta delle parti e il giudizio abbreviato, fortemente incentivati dopo la riforma del codice, contribuiscono ad alleggerire il carico dei dibattimenti penali, ma in misura non comparabile a quanto avviene nei sistemi inglese e degli Stati Uniti, dal momento che statisticamente non superano il trenta per cento del totale.

Fra gli interventi destinati ad evitare la celebrazione del dibattimento va ora annoverato anche l'istituto della sospensione del 
procedimento con messa alla prova ${ }^{31}$, già sperimentato nel processo minorile ed introdotto in quello ordinario dalla sopra citata legge n. 67 del 2014. Per i reati puniti con pena detentiva non superiore nel massimo a quattro anni, è applicabile, su richiesta dell'imputato, un programma di trattamento a fini di risocializzazione, il cui esito positivo estingue il reato (artt. 168-bis-168-quater c.p.). La sua attivazione già nella fase delle indagini preliminari consente una notevole economia processuale, evitando di regola l'esercizio dell'azione penale e la celebrazione del giudizio. La semplificazione si risolve pertanto in una procedura di tipo prevalentemente burocratico-amministrativo, sebbene formalmente giurisdizionale. La fuga dal processo per i reati meno gravi - che comunque statisticamente sono la maggioranza - si giustifica in nome dell'efficienza complessiva del sistema: ma non è detto che la sospensione con messa alla prova sia necessariamente appetibile dall'imputato, dal momento che per la medesima fascia di reati esistono soluzioni alternative che possono in concreto rivelarsi anche più vantaggiose.

Occorre pertanto spingersi oltre nella razionalizzazione del sistema, agendo sui presupposti e sulle modalità di esercizio dell'azione penale. La razionalizzazione è solo un passo avanti verso l'efficienza, ma non è certamente il passo risolutivo. Tuttavia se a ciò si accompagnano altri interventi, di carattere strutturale, e soprattutto la massimizzazione delle risorse disponibili - non tanto e non solo l'aumento, pure necessario, quanto una loro migliore allocazione - le ricadute positive, almeno nel medio periodo, potrebbero manifestarsi anche nei giudizi più complessi e per i reati più gravi, recuperando l'impostazione originaria del processo accusatorio.

\section{Biblografia}

K. AMBOS, International criminal procedure: "adversarial", "inquisitorial" or mixed?, in Int'l. Crim. L. Rev., 2003, p. 1 s. https://doi.org/10.1163/156753603767877084

31 In argomento, e sui dubbi di illegittimità costituzionale per contrasto con la presunzione d'innocenza, v. R. Orlandi, Procedimenti speciali, in Compendio di procedura penale, a cura di G. Conso, V. Grevi, M. Bargis, 6. ${ }^{\text {a }}$ ed., Cedam, Padova, 2014, p. 746 s.; C. Cesari, sub art. 464-bis, in Commentario breve al codice di procedura penale, a cura di G. Conso e G. Illuminati, 2. ${ }^{\text {e }}$ ed., Cedam, Padova, 2015, p. 2124. 
M. CAIANIELLO, Disclosure before the ICC. The emergence of a new form of policies implementation system in international criminal justice?, in Int'l Crim. L. Rev., 2010, p. 23 s. https://doi.org/10.1163/157181209x12584562670776

G. CARMIGNANI, Teoria delle leggi della sicurezza sociale, Pisa, 1832, IV, 46 s.

C. CESARI, sub art. 464-bis, in Commentario breve al codice di procedura penale, a cura di G. Conso e G. Illuminati, 2. ${ }^{\text {a }}$ ed., Cedam, Padova, 2015, p. 2124.

M. CHIAVARIO, Ancora sull'azione penale obbligatoria: il principio e la realtà, in Id., L'azione penale tra diritto e politica, Padova, 1995, p. 131.

A. CIAVOLA, Il contributo della giustizia consensuale e riparativa all'efficienza dei modelli di giurisdizione, Torino, 2010, p. 95 s.

G. CONSO, Accusa e sistema accusatorio (diritto processuale penale), in Enc. dir., I, Milano 1958, p. 336.

F. CORDERO, Procedura penale, 9a ed., Milano 2012, p. 21-38.

M.R. DAMAŠKA, Evidence Law Adrift, New Haven-London, 1997, trad. it., Il diritto delle prove alla deriva, Bologna, 2003, p. 113 s.

V., FANCHIOTTI, The right to a speedy trial nell'esperienza nordamericana, in AA.VV., La ragionevole durata del processo. Garanzie ed efficienza della giustizia penale, a cura di R.E. Kostoris, Torino, 2005, p. 13 s.

L. FERRAJOLI, Diritto e ragione. Teoria del garantismo penale, 8a ed., Bari, 2004, p. $574 \mathrm{~s}$.

P. FIORELLI, Accusa e sistema accusatorio (diritto romano e intermedio), in Enc. dir., I, Milano, 1958, p. 330-331.

G. FOSCHINI, Sistema del diritto processuale penale, I, Milano, 1965, $226 \mathrm{s.}$

C. F. GROSSO, La non punibilità per particolare tenuità del fatto, in Dir. pen. proc., 2015 , p. 517.

G. ILLUMINATI, El sistema acusatorio en Italia, in AA.VV., Proceso penal y sistemas acusatorios, a cura di L. Bachmaier Winter, Madrid, 2008, p. 135 s.

J. H. LANGBEIN, Torture and plea bargaining, in 46 Univ. Chicago L. Rev., 197879, p. 3 s. https://doi.org/10.2307/1599287

L. LUCCHINI, Elementi di procedura penale, Firenze, 1895, p. 19.

A., NAPPI, Guida al codice di procedura penale, 10a ed., Milano, 2007, p. 13 s.

D. NELKEN, La durata del processo penale in prospettiva empirica: riforme giuridiche e contesti sociali, in AA.VV., La ragionevole durata del processo. Garanzie ed efficienza della giustizia penale, a cura di R.E. Kostoris, Torino, 2005, p. 28. 
R. ORLANDI, Procedimenti speciali, in Compendio di procedura penale, a cura di G. Conso, V. Grevi, M. Bargis, 6. ${ }^{\mathrm{a}}$ ed., Cedam, Padova, 2014, p. 746 s.

F. PALAZZO, Nel dedalo delle riforme prossime e venture, in Riv. it. dir. e proc. pen., 2014, p. 1706 s.

M. PLOSCOWE, The development of present-day criminal procedures in Europe and America, in 18 Harv. L. Rev., 1935, p. 453 s., 459-60. https://doi. org/10.2307/1332766

G. PUGLIESE, Processo privato e processo pubblico, in Riv. dir. proc., 1948, p. 72 s.

L. RADZINOVICZ, A History of English Criminal Law and its Administration from 1750, I, London, 1948, p. 3 s.

R. VAN CLEAVE, An offer you can't refuse? Punishment without trial in Italy and the United States: the search for truth and an efficient criminal justice system, in 11 Emory Int'l L. Rev., 1997, p. 419 s.

D. VICOLI, Scelte del pubblico ministero nella trattazione delle notizie di reato e art. 112 Cost: un tentativo di razionalizzazione, in Riv. it. dir. proc. pen., 2003, p. 251 s.

\section{Informações adicionais e declarações dos autores (integridade científica)}

Declaração de conflito de interesses (conflict of interest declaration): o autor confirma que não há conflitos de interesse na realização das pesquisas expostas e na redação deste artigo.

Declaração de coautoria e especificação das contribuições (declaration of authorship): todas e somente as pessoas que atendem os requisitos de autoria deste artigo estão listadas como autores; o autor se responsabiliza integralmente por este trabalho em sua totalidade.

Declaração de ineditismo e originalidade (declaration of originality): este artigo é uma versão revisada e ampliada do trabalho publicado em: G. Illuminati, El sistema acusatorio en Italia, in AA.VV., Proceso penal y sistemas acusatorios, a cura di L. Bachmaier Winter, Madrid, 2008, p. 135 s.; o autor assegura que não há plágio de terceiros. 
Dados do processo editorial

(http://www.ibraspp.com.br/revista/index.php/RBDPP/about/editorialPolicies)

- Recebido em: 24/04/2018

Equipe editorial envolvida

- Retorno rodada de correções: 26/05/2018

- Autor convidado

- Editor-chefe: 1 (VGV)

http://www.ibraspp.com.br/revista/index.php/

- Editoras-associadas: 2 (LBW e BC)

RBDPP/about/editorialPolicies - custom-1

\section{COMO CITAR ESTE ARTIGO:}

ILLUMINATI, Giulio. Modello processuale accusatorio e sovraccarico del sistema giudiziario. Revista Brasileira de Direito Processual Penal, Porto Alegre, vol. 4, n. 2, p. 533-557, mai./ago. 2018.

https://doi.org/10.22197/rbdpp.v4i2.164

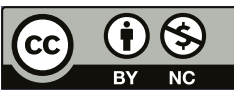

Esta obra está licenciada com uma Licença Creative Commons Atribuição-NãoComercial 4.0 Internacional. 